\title{
Outreach Activities in High Energy Physics
}

\author{
Kétévi Adiklè Assamagan ${ }^{1}$ \\ Brookhaven National Laboratory \\ Department of Physics, Upton, New York, 11983, USA \\ E-mail: keteviabnl.gov
}

There are many activities in high energy physics for education, communication and outreachthe ECOsystem - to raise public awareness and support, motivate learners to develop and maintain interest, educate students, promote inclusion and diversity, and demonstrate the impact of high energy physics research. Public support and coordination of ECO are needed at all levelsregional, national and international. I will discuss a few examples of ECOsystem in high energy physics, with a particular focus on outreach activities in Africa.

XXIX International Symposium on Lepton Photon Interactions at High Energies - LeptonPhoton2019 August 5-10, 2019

Toronto, Canada

\section{${ }^{1}$ Speaker}




\section{1. Introduction}

Outreach activities engage professional educators, communicators and physicists. The educators help people acquire knowledge, competence and confidence. The communicators advise on management, do strategic planning, and monitor opportunities, to help disseminate scientific knowledge. The physicists carry out the scientific research; they may also be teachers or participate in outreach activities. In addition, physicists engage decision makers in matters of education and society in general. To ensure success, the efforts of all players-educators, communicators and physicists - should be coordinated at regional, national and international levels and generate public support. Success also requires human and financial resources. However, resources dedicated to ECO vary across countries. An international agreement on a minimal support dedicated to ECO might be useful for concerted resource allocations to these efforts. We also need to demonstrate the economic, social and environmental impacts of particle physics research; this may facilitate support from third-party advocates. In these ECO efforts, public perceptions of physicists count, e.g. "What does a physicist look like?", so do inclusion and diversity. Although there has been progress, diversity in our field still needs improvement [1].

After the discovery of the Higgs boson, the high energy physics community (HEP) designed many more outreach activities for an enlarged audience. To list a few: masterclasses with real Higgs boson data, CERN sixtieth anniversary, beamline for school, high school pupils and teachers' program, open days at CERN and European labs, traveling and collider exhibitions, public event during conferences, dark matter days, HL-LHC media, and CERN science gateway expected in 2022 [1].

In this note, I will discuss a few examples of successful outreach activities, namely the international masterclasses and the phantom of the universe. Then, I will touch upon some of the ECOsystems in the Africa, such as the African Institute for Mathematical Sciences (AIMS) and the African School of Fundamental Physics and Applications (ASP).

\section{International masterclasses}

A masterclass consists of introductory lectures to HEP, virtual tours of the LHC experiments, analyses of authentic HEP data and videoconferences with physicists. For the participants, the masterclass instills some understanding about particle physics research. The masterclasses have grown to include upwards of fifty countries and ten thousand participants as shown in Figure 1. The scientific program is based on the four major experiments at the LHC (ATLAS, CMS, ALICE and $\mathrm{LHCb}$ ), and expanded to include neutrino and particle therapy masterclasses. New measurements based on the CMS upgrade and the Belle II experiment are also added [2].

The masterclasses offer new opportunities, namely the Worldwide Data Day (W2D2) and International Day of Women and Girls in Science (IDWGS). The W2D2 consists of simple measurements of azimuthal and polar angles of muons in ATLAS or CMS events. It is organized each year in October or November. The IDWGS is based on LHC masterclass measurements, done by girls, to learn about particle physics and discuss women in science. It takes place yearly in February.

The masterclasses are expanded with new measurements-based on CMS four-lepton events, Belle II B-physics analysis, MINERvA and MicroBooNE, particle physics therapy, and heavy ions. The expansion offers a rich variety in the analysis methods presented to the participants. The masterclasses are growing with the addition of more countries and an extension in the scientific program, with more centers for videoconferences. The growth is a sign of a positive impact of the program; however, such a growth also comes with challenges to adapt the 
structure, organization and coordination. The organizers encourage interested colleagues to reach out and get involved [2].

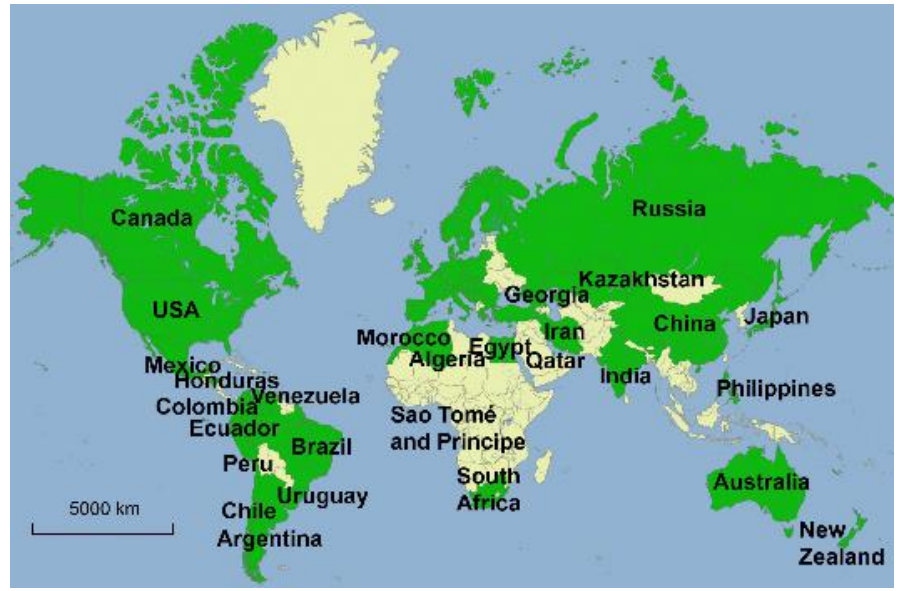

Figure 1: Countries that participated in the international masterclasses in 2019. The efforts of AIMS and ASP, as discussed in Sections 4 and 5, could improve the coverage of international outreach in Africa.

\section{3. Phantom of the universe}

A planetarium show about dark matter, called the "Phantom of Universe", covers astrophysics, an underground experiment and the LHC [3]. It has been translated into twenty-one languages and seen by about four million people in five hundred and ten planetariums in sixty-six countries. The show is distributed to the planetariums for free, and presented for months at a time. A planetarium show is dramatic, and constitutes a great way to show galaxies, particle collisions, etc. Planetariums have the perfect science-interested audience, i.e. the general public and K12; over one hundred million people a year watch planetarium shows. The authors of the show solicited the expertise of an international team of professionals, including an Academy Awardwinning actress as the narrator, Academy award winning sound by Skywalker Sound, and a Hollywood scriptwriter and producer [3].

\section{4. AIMS}

The African Institute for Mathematical Sciences is a pan-African network of centers of excellence to train African students with the objective to prepare the next generation of scientists for sustainable development [4]. It offers a one-year intensive MSc program to African students in mathematical physics and applications, in addition to doctoral training and post-doctoral fellowships. The first AIMS center opened in Cape Town, South Africa in 2003. Since then, through the AIMS Next Einstein Initiative (AIMS-NEI), AIMS centers have opened in Senegal (2011), Ghana (2012), Cameroon (2013), Tanzania (2014) and Rwanda (2016). The goal is to have fifteen AIMS centers of excellence in operation across Africa by 2023 [4]. AIMS has produced upwards of one thousand two hundred graduates, of whom thirty-one percent are women and sixty-five percent are working in Africa. AIMS has chapters in Canada, Germany and the United Kingdom; the chapters drive partnerships for resource mobilization and public engagement to support the growth of the AIMS network.

\section{5. ASP}

The African School of Fundamental Physics and Applications is a biennial school in Africa [5]. It is based on the observation that fundamental physics provides excellent motivation for students of science. The aim of the school is to build capacity to harvest, interpret, and exploit the results of current and future physics experiments and to increase proficiency in related applica- 
tions. The participating students are selected from all over Africa. The school also offers a workshop to train high school teachers, outreach to motivate high school pupils and a physics conference to support a broader participation of African research faculties. Support for the school comes from institutes in Africa, Europe, USA and Asia. The first school took place in Stellenbosch, South Africa on August 1-21 2010, the second edition in Kumasi, Ghana on July 15-August 8 2012, the third edition in Dakar Senegal on August 3-23 2014, the fourth biennial school at the University of Rwanda on August 1-19 2016, and the fifth edition in Namibia on June 24-July 14 2018. The next edition of the school is planned in 2020 in Morocco [5].

\subsection{Introduction}

The basic objective is to help improve the quality of higher education in Africa and increase the number of African students acquiring higher education. This is achieved through an outreach effort, an increased awareness of the potential of high-quality training offered by large scale experiments in context of various scientific disciplines, and a system of networking on the international scale. There is a strong alignment between the mission and the vision of African governments and policy makers on education and capacity building and their programs with the goals of the ASP. The ASP is committed to include African governments in the planning, in order to take advantage of aspects such as consolidating agreements and their goals, building on synergy with other programs, improving the sustainability and impact of capacity development and improving the measurement and visibility of the impact. By working with African governments and policy makers on education, ASP seeks to promote a culture of science that creates an attractive environment for African student alumni, thus encouraging their retention within Africa. ASP promotes sustainable scientific development in Africa by building a network between African and international researchers for increased collaborative research and shared expertise.

The schools are based on a close interplay between theoretical, experimental, applied physics, and Grid computing. They cover a wide range of topics: particle physics, particle detectors, astro-particle physics and cosmology, computing, accelerator technologies, medical physics, condensed matter, light sources and their applications. Scientists from Africa, Europe, Asia and the USA are invited to prepare and deliver lectures according to the proposed topics considering the diverse levels of the students. The duration of the school allows for networking - interactions among students and between students and lecturers. The schools are funded by institutes in Africa, Asia, Europe and the USA. ASP serves at least two purposes: the organization of the school creates understanding of the many challenges and provides a template for solving them, and secondly it provides opportunity to prepare the students to find practical answers to many issues that they face.

\subsection{Venue and scope}

The school occurs biennially in different African countries, taking advantage of local support and considering a uniform exposure for Africa. The proposed duration of the school is three weeks. The host country of the school is selected two and a half years in advance through a competitive bidding process that considers safety of the participants and the support from the host country government. The target - in each edition of the ASP - is to have eight-five students selected from five hundred applicants in various African countries, seventy high school teachers from the host country for a one-week teachers training workshop, one thousand five hundred high school pupils for a one-week outreach, and sixty extra participants at the ASP conference. The objective of the ASP conference is to attract the participation of ASP alumni and African research faculties to give scientific talks and network with the international participants to foster new research collaborations. Peer-reviewed conference proceedings are published in the African Review of Physics. Full bursaries are provided to the selected students. Figures $2-5$ show the distributions of the selected students as a function of their citizenships, age and field of study. Alumni are not 
re-selected for the current school but they may attend the conference with the support of their academic institutes.

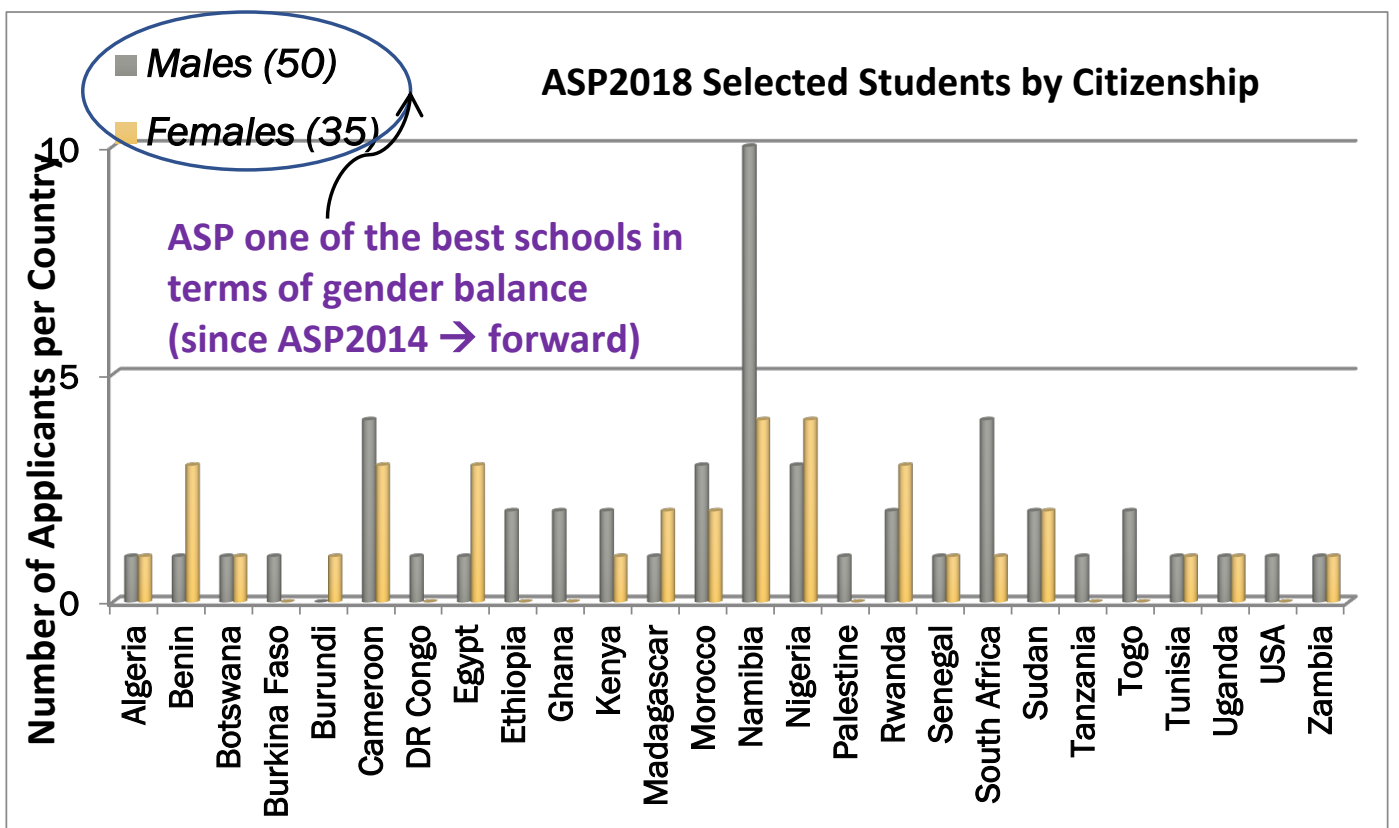

Figure 2: Distribution of ASP2018 selected students by citizenship. There were 523 applicants from whom 85 were selected. There were an additional 30 good students on the waiting list. The selection is constrained by budget and logistics, and all the early declinations are replaced from the waiting list.

\subsection{Coordination of the activities}

ASP activities will be coordinated by the International Organizing Committee (IOC) in collaboration with a Local Organizing Committee (LOC) in the host country. The IOC is advised by an International Advisory Committee (IAC). International lecturers (IL) help design the scientific program, which is often adapted to reflect the research interests of the host country. The lecturers also help in the selection and mentorship of the students. The ASP mentorship program is a dedicated effort to mentor ASP students in collaboration with their academic advisors; the mentorship program runs continuously even when there is no formal school.

In the addition to the coordination of all the activities, the IOC is responsible for fundraising, identification of the next host country, and the activity reports to the funding agencies. Each funding agency has up to two representatives in the IAC to advise on the usage of the funds, the selection of the next host country, and on the rest of the activities. The LOC helps with all the local logistics and liaises with the education and research branches of the host country government. 


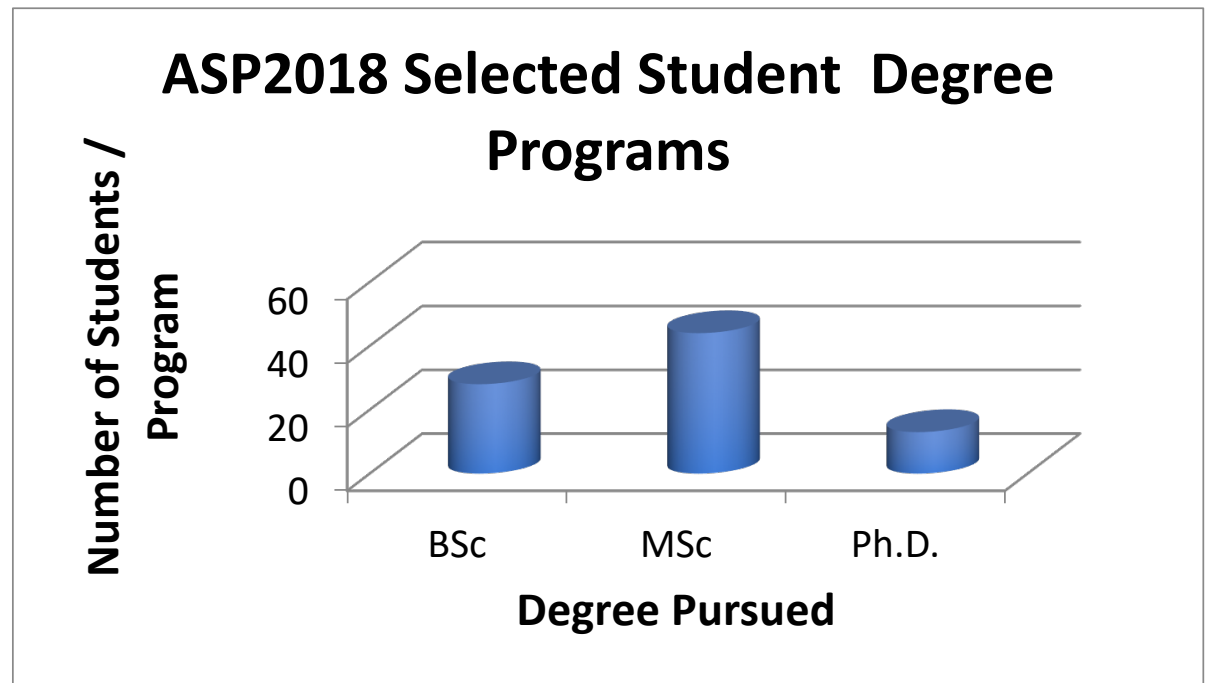

Figure 3: The academic levels of the ASP2018 students at the time of the selections. Participating students are mostly at the MSc level.

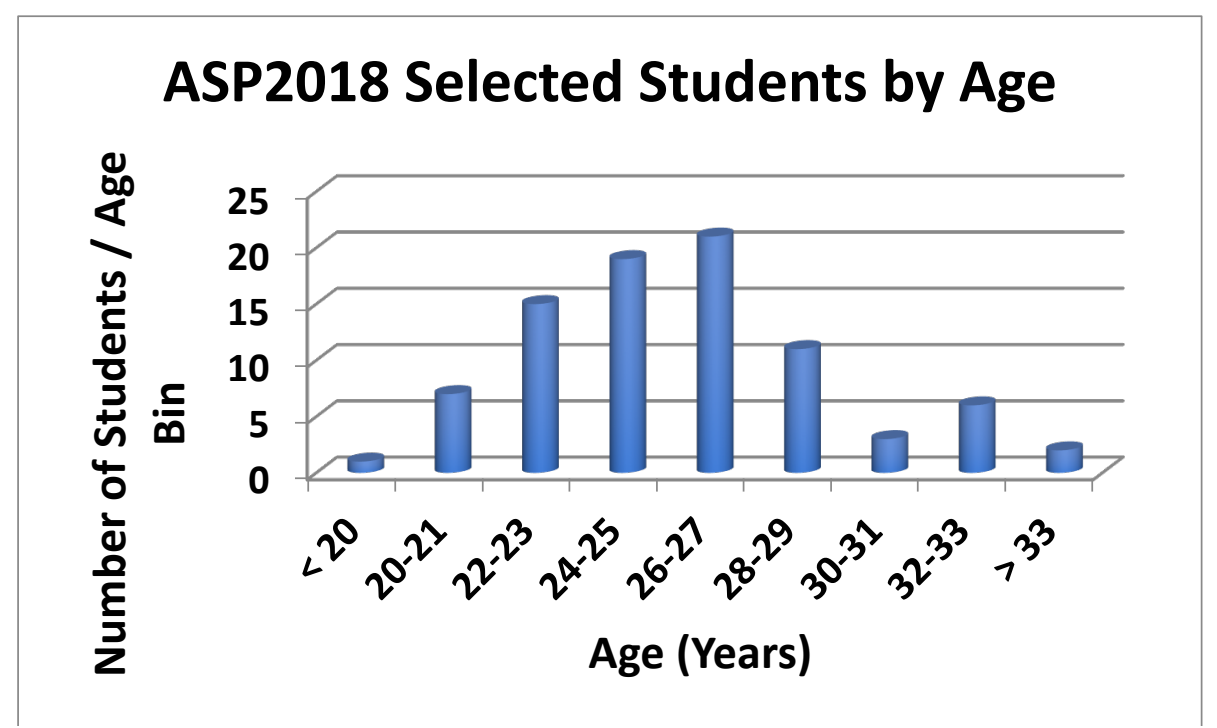

Figure 4: The age distribution of the selected students. The peak is around 26-27 years old with the academic level at the MSc. 


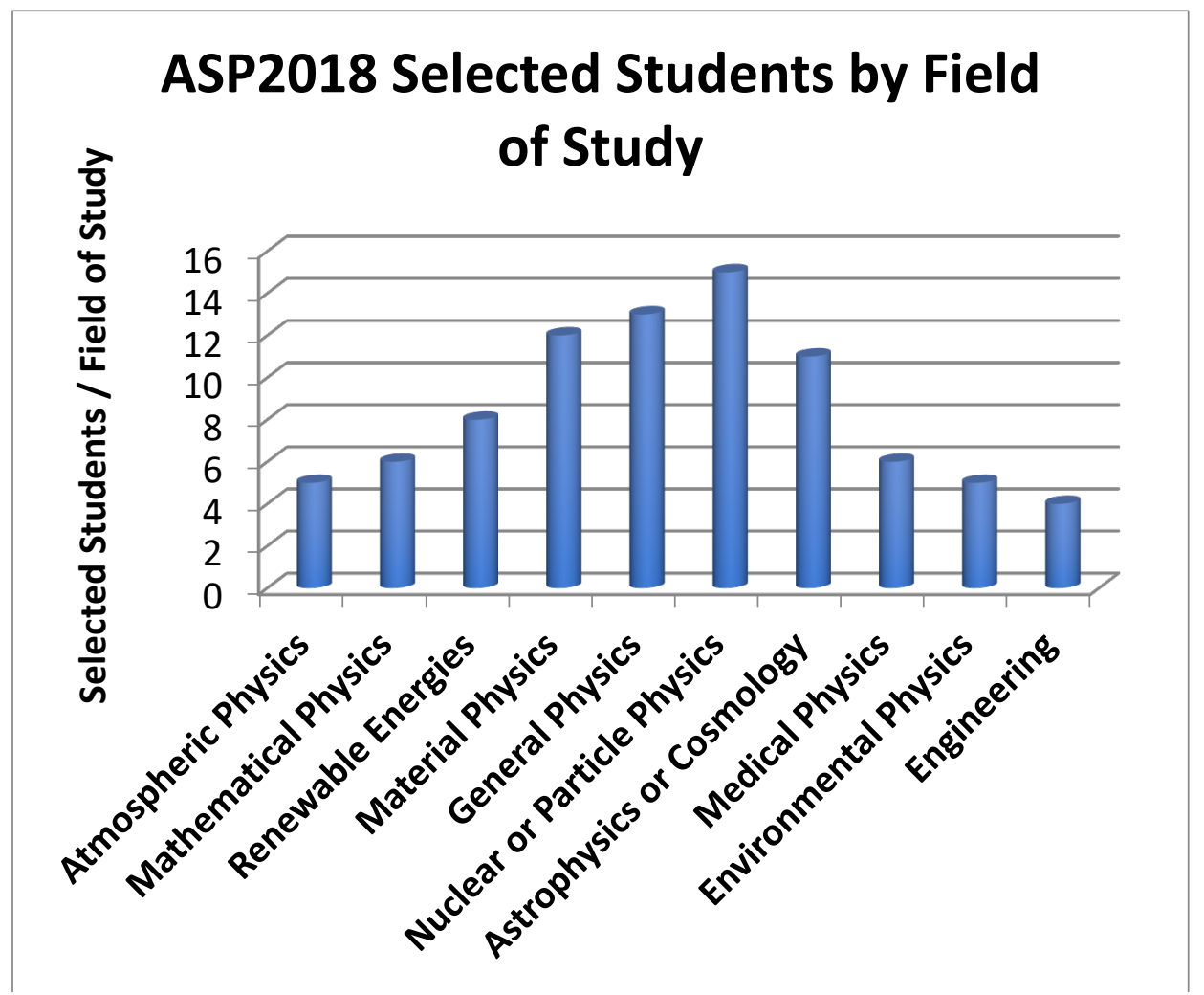

Figure 5: The field of study of the ASP2018 students at the time of their selection. Fifteen to twenty percent of the selected students have their majors in particle physics.

\subsection{Impact and retention}

Two types of surveys are done about ASP. First, toward the end of the current edition of the school, the participating students and teachers are asked for feedback on their experience. The second survey is conducted every four years on all the alumni- this allows the IOC to follow the academic development of the alumni and answer questions such as, "What happens to the students after they have attended ASP?" or "Where are the students now?" The continuous mentorship program also helps answer these questions since the mentors follow the academic development of the students. Figures 6-8 show some feedback from the students and high school teachers.

\subsection{Outlook}

The success of ASP is also due to the dedication of the international lecturers, most of whom cover their travels from external sources. This allows the funds raised by the IOC to be used to maximize the attendance of African students. Although the school duration is three weeks, most lecturers stay for only one of the three weeks on average to share their experience with African students. Some of the lecturers identify and help students pursue high education or research at their institutes.

One day during the school, the IOC organizes a forum on capacity development and retention in Africa. This forum provides the platform to engage African policymakers on physics education and research, the media, the industries and the international community in a discussion about the role of the ASP. The main objective of the forum is to improve the ASP program to serve the education and research priorities of African countries. 


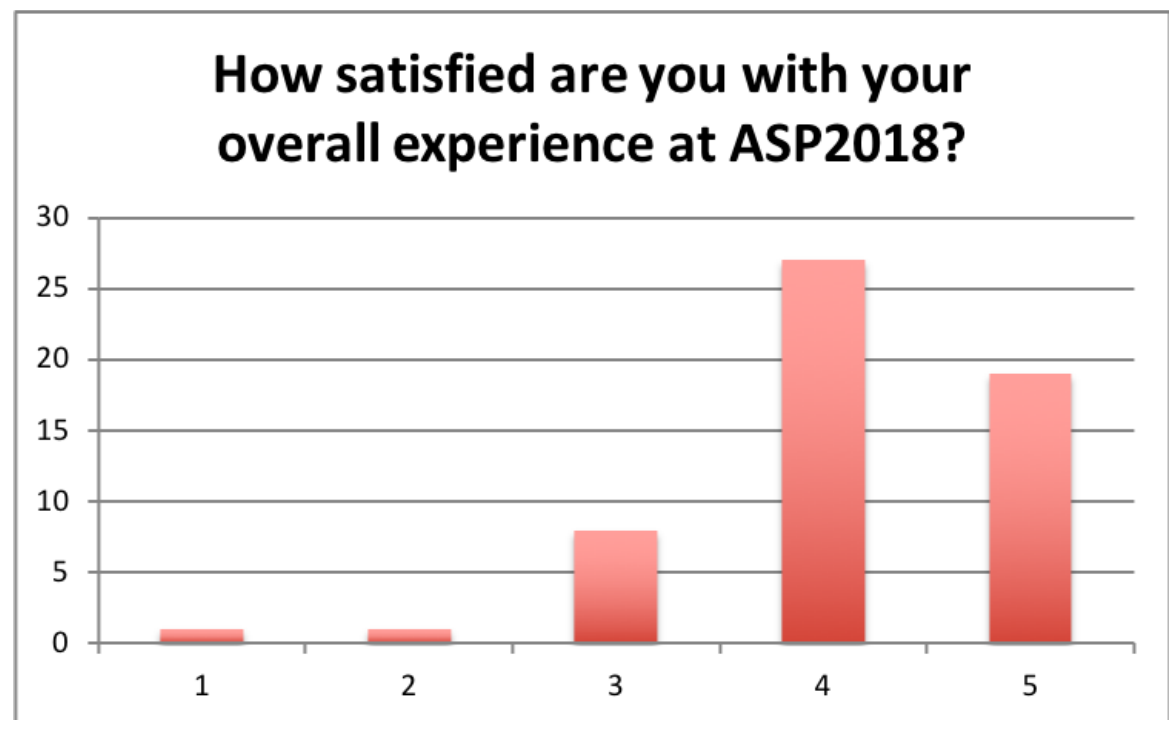

Figure 6: students" satisfaction about their experience at ASP2018. "1" is the least satisfied and " 5 " shows the most satisfaction.

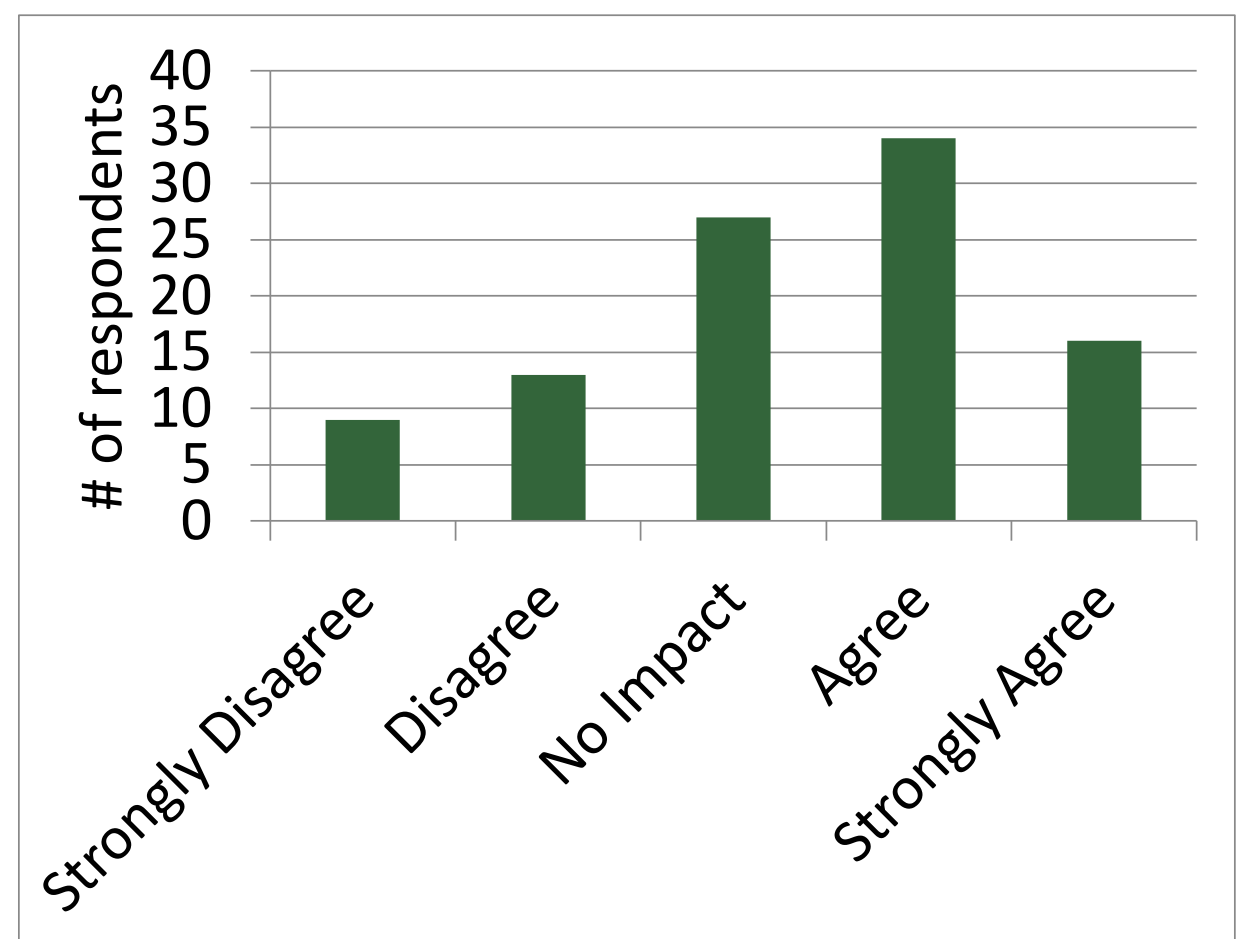

Figure 7: The alumni were asked if their research has benefited from professional contacts made at ASP — during the school or afterward through mentorship. 


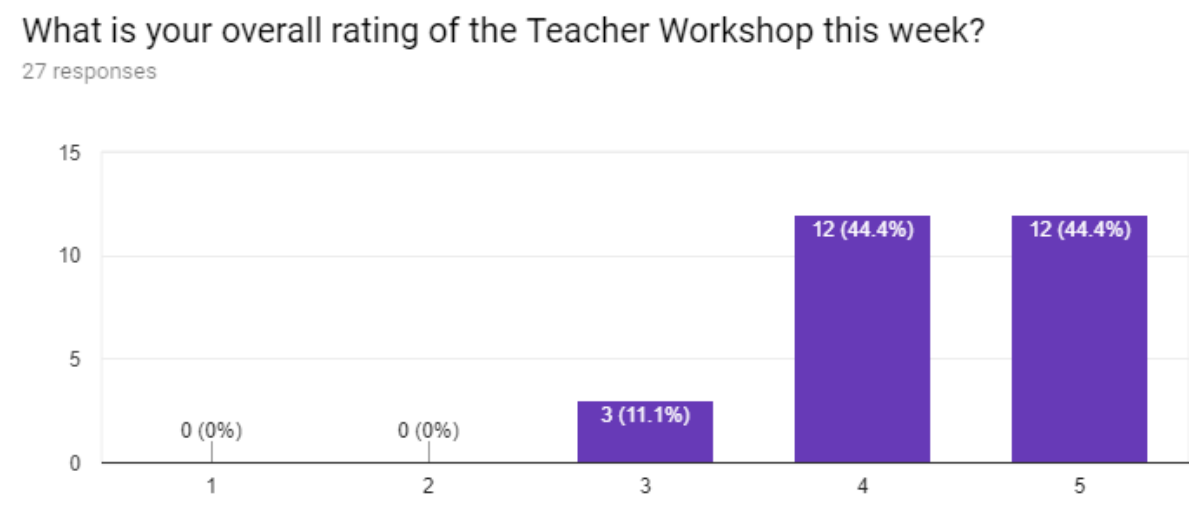

Figure 8: Feedback from the high school teachers on their experience during the workshop for teachers at the ASP2018 in Namibia.

The ASP forum also encourages the promotion of research collaboration and consortia, e.g. ASP served as the platform for earlier discussions on the African Light Source [6]. Through the ASP forum, collaboration among African countries are encouraged to share experience and approaches, and find concerted solutions to common issues in education.

The issues of retention - within Africa - of the qualified African graduates are also debated during the forum. The survey results show that most of ASP alumni stay in Africa-only $20 \%$ go outside for high education and research. The objective of the discussions on retention is to develop a mechanism to reintegrate into the Africa the alumni that go abroad.

\section{6. Conclusions}

6. There are many successful ECOsystems in HEP. The key is to coordinate ECO efforts at all levels, and demonstrate the impact of HEP, while improving inclusion and diversity. Improvement of ECO is much needed in Africa. There, progress can be made through the support of the well-established AIMS and ASP. The ASP has evolved to be much more than a school. It is a program of actions with directed ethos toward physics as an engine for development in Africa.

\section{References}

[1] P. Royole-Degieux, Education, Communication and Outreach; it's essential, Open symposium European Strategy for Particle Physics, https://indico.cern.ch/event/808335/.

[2] K. Cecire, U. Bilow. International Masterclasses, www.physicsmasterclasses.org/.

[3] M. Barnett, K. De, R. Schwienhorst, Phantom of the Universe, http://PhantomOfTheUniverse.org/.

[4] N. Turok, The African Institute for Mathematical Sciences, https://www.nexteinstein.org/.

[5] K.A. Assamagan, B. Acharya, A.E. Dabrowski, C. Darve, J.R. Ellis, F. Ferroni, S.G. Muanza, The African School of Fundamental Physics and Applications, https://www.africanschoolofphysics.org/

[6] S.H. Connell et. al, The African Light Source Project, in proceedings of the first African Conference on Fundamental Physics and Applications, The African Review of Physics (2018) 13: 0019. 